\title{
Dynamic optical coherence tomography of skin blood vessels - proposed terminology and practical guidelines
}

\author{
M. Ulrich, ${ }^{1, *}$ L. Themstrup, ${ }^{2}$ N. de Carvalho, ${ }^{3}$ S. Ciardo, ${ }^{3}$ J. Holmes, ${ }^{4}$ R. Whitehead, ${ }^{4}$ J. Welzel, ${ }^{5}$ \\ G.B.E. Jemec, ${ }^{2}$ G. Pellacani ${ }^{3}$ \\ ${ }^{1} \mathrm{CMB}$ Collegium Medicum Berlin, Berlin, Germany \\ ${ }^{2}$ Department of Dermatology, University of Copenhagen, Roskilde Hospital, Roskilde, Denmark \\ ${ }^{3}$ Department of Dermatology, University of Modena and Reggio Emilia, Modena, Italy \\ ${ }^{4}$ Michelson Diagnostics, Maidstone, UK \\ ${ }^{5}$ Department of Dermatology, Klinikum Augsburg, Augsburg, Germany \\ ${ }^{\star}$ Correspondence: M. Ulrich. E-mail: m.ulrich@collegiummedicum.de
}

\begin{abstract}
Background Dynamic optical coherence tomography (D-OCT) has recently been introduced in dermatology. In contrast to 'Standard' OCT imaging, which exclusively relies on the morphological analysis of the tissue, D-OCT allows the in vivo visualization of blood flow. Preliminary D-OCT data showed differences in the vascularization of nevus to melanoma transition, suggesting that this technology may help to differentiate between benign and malignant lesions.

Objective Several factors may influence the quality of D-OCT imaging. Therefore, standard operating procedures as well as a common terminology are required for better validation and comparison of the images.

Methods Here, we present practical guidelines for optimal image acquisition and a proposed terminology on vascular patterns observed by D-OCT.

Results Dynamic OCT allows the morphologic distinction of different vascular shapes (e.g. dots, blobs, curves, lines), their distribution and organization within skin lesions.
\end{abstract}

Conclusion D-OCT adds functional information on skin microvasculature and the vascular networks within lesions.

\section{Conflicts of Interest}

MU: stakeholder in CMB Collegium Medicum Berlin GmbH; clinical trials for Almirall, Biofrontera, Leo Pharma and Novartis; paid lectures for Almirall, Biofrontera, Galderma, Leo Pharma, Mavig GmbH, Michelson

Diagnostics.

$\mathrm{JH}$ and RW are employees of Michelson Diagnostics.

GBE Jemec has received honoraria from AbbVie, MSD, and Pfizer for participation on advisory boards, and grants from Abbvie, Actelion, Janssen-Cilag, Leo Pharma, Novartis and Regeneron for participation as an investigator and received speaker honoraria from AbbVie, Galderma, Leo Pharma and MSD. He has furthermore received unrestricted research grants from AbbVie and Leo Pharma.

\section{Funding sources}

This work is part of an EU-funded project (ADVANCE, Project Number 621015).

\section{Introduction}

Optical coherence tomography (OCT) represents a non-invasive imaging technique that has recently showed its applicability for the diagnosis of non-melanoma skin cancer and especially basal cell carcinoma. ${ }^{1-4}$ Although different devices are available, which show variations in some technical parameters, 'standard' OCT generally relies on the morphological analysis of the tissue. ${ }^{5,6}$ As a recent technical improvement, 'dynamic' OCT (D-OCT) allows the visualization of blood flow in vivo and therefore enables the visualization of microvasculature..$^{2,7-10}$ As in all imaging techniques, correct image acquisition is important in D-OCT, but as functional data of blood flow is produced, special attention to the acquisition process and the reproducibility of the findings is required.

New technology often needs new terminology, which is of major importance for the description of findings, for interpretation of data and to extract information. This manuscript presents the first expert suggestions on standard operating procedures in D-OCT as well as a proposal for common D-OCT terminology. 


\section{Materials and methods}

The work presented herein is part of an EU-funded project (ADVANCE, Project No. 621015). The technical partner (Michelson Diagnostics Ltd) was responsible for development of the equipment and for technical support. Four clinical partners acquired data from normal skin as well as from several skin diseases. These images were reviewed, resulting in the development of a standard operative procedure for optimal imaging acquisition and a suggested terminology for D-OCT based on the experience of the authors. The protocol has been approved by the local ethical committees, and informed consent was obtained prior to enrolment. All research was conducted according to the declaration of Helsinki principles. Four identically configured VivoSight 'Dx' dermatological OCT scanners equipped with 20-kHz swept-source laser and 'Dynamic OCT' processing and software (Michelson Diagnostics, Kent, UK, www.vivosight.com) were used.

\section{Imaging process}

The hand-held probe VivoSight 'Dx' dermatological OCT scanner provides a $6 \times 6 \mathrm{~mm}$ field of view, showing vertical- and horizontal-plane OCT images, with a depth penetration of $1 \mathrm{~mm}$. Vascular imaging is usually limited to $<500 \mu \mathrm{m}$ because of the speckle noise/signal ratio in the deeper parts of the skin. Image acquisition does not require contact gel. The distance between the probe lens and the skin is ensured by a spacer engaged with the head of the probe and in contact with the skin to stabilize image acquisition. The dynamic OCT data are collected simultaneously with the structural OCT data and are immediately displayed on the structural OCT images as an overlay, so that the relationship between the vessels and structures can be seen. Areas of blood flow are displayed as red. Typically, a 'stack' of 120 OCT images is captured for each lesion to form a $3 \mathrm{D}$ 'data block' in approximately 10-20 s. This can then be simultaneously viewed from the side ('B-scan', cross-sectional) or from the top ('en-face') at any desired depth. En face images are the most useful for the study of microvasculature patterns.

For the acquisition of D-OCT images, the probe should be positioned on the skin without any pressure in order to avoid compression of the vessels, which would lead to a reduction in blood flow velocity. During the imaging process, the probe needs to be held in a stable position parallel to the skin surface. This may be obtained by holding the probe spacer between thumb and index finger of one hand and light support of the probe body with the other hand.

On the computer screen, target marks indicate the optimal focus area, and the image should be positioned with the epidermal surface aligned with the top of the target area (Fig. 1).

\section{Terminology}

When analysing D-OCT images, several factors have to be considered in addition to the morphology of the vessels. Vascular pattern is assessed in en face images.

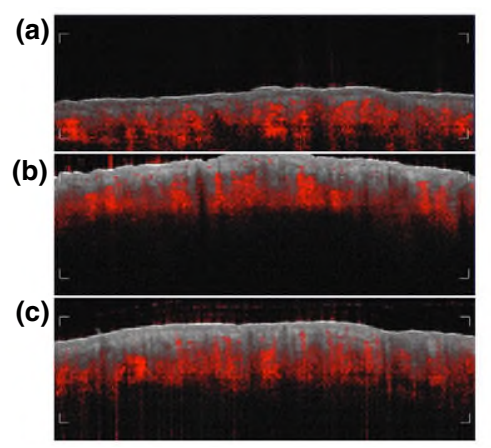

Figure 1 It shows the optimal positioning of the images within the target marks. (a) illustrates an incorrect image acquisition with the OCT image positioned too low. (b) shows an incorrect image acquisition with the OCT image being positioned too high. (c) illustrates the correct image acquisition with the OCT image being positioned correctly with the top surface aligned with target marks.

En face image parameters We propose a two-step evaluation, which includes the global analysis of pattern architecture (D-parameters) and the description of specific shapes of vessels (S-parameters).

The D-parameters include the depth, the density, the diameter, the direction and the distribution of the vessels on the scan.

Depth The depth is calculated from the outer epidermal layer automatically identified using dedicated software, which reconstructs en face images with the points at the desired tissue depth, following the epidermal contour. ${ }^{9}$ En face images at predetermined depths can be rapidly extracted by the dedicated software. After evaluation of en face scans in normal skin and different tumour types (i.e. melanoma, basal cell carcinoma, squamous cell carcinoma, nevi and seborrhoeic keratosis), it has been proven useful to perform the analysis of en face images at three different standard depths of 150, 300 and $500 \mu \mathrm{m}$. In these standardized depths, the capillaries in the dermal papillae as well as the superficial and deep dermal plexus are displayed. Other depths can be included as needed. When different skin sites are compared or a lesion is monitored over time, it should be assured that the horizontal OCT images are from the same depth.

Density The density of the vessels can be assessed by visually comparing against reference images with different vessel densities (Fig. 2).

Diameter The use of a reference image is proposed, and the scoring should be performed as 'average', 'less' or 'more' compared to the reference image (Fig. 2).

Instead of comparing with standard reference images, both parameters can be assessed by comparison between diseased skin and adjacent or opposite healthy skin, serving as an internal 

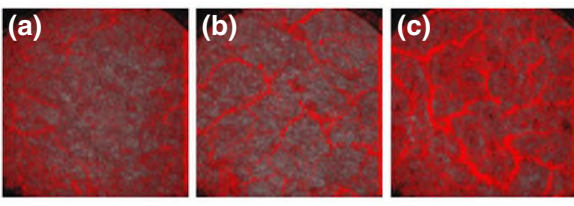

Figure 2 Diameter and density of vessels in reference images of healthy skin, left image: low, centre image: average, right image: high.

control. This procedure respects location-dependent and individual differences as well.

Direction Vessels can either be oriented parallel to each other (streaming) or radiating in all directions from a central point, or do not show any direction at all.

Distribution The distribution of the vessels may be regular (the same pattern and aspect throughout the imaged area), clustered (the presence of areas with a group of vessels with the same aspect) or irregular (chaotic distribution and different aspects of vessels).

The S-parameters focus on the analysis of the single vessels in the en face images resulting in the description of six different shapes of vessels being present in our large set of lesions. These

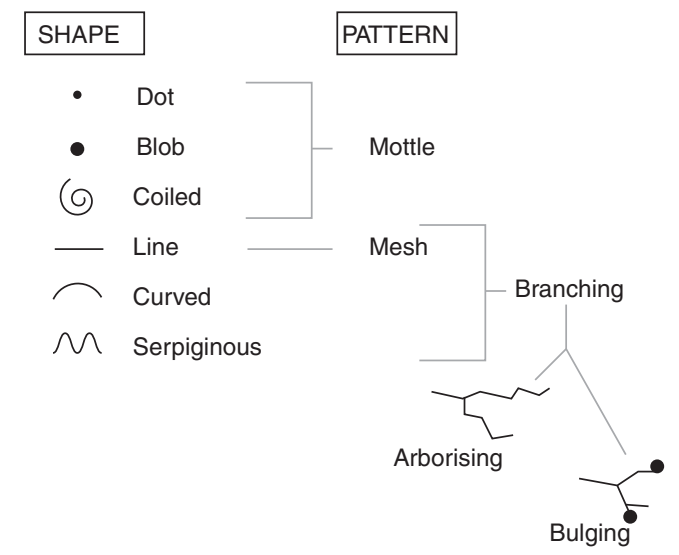

Figure 3 It shows a schematic illustration of the different shapes of vessels being observed on D-OCT as well as different patterns that can be observed within a lesion.

'Shapes' include dots (small red points corresponding to vessels oriented vertical to the surface), blobs (larger round to oval red globules corresponding to vessels oriented vertical to the surface), coiled vessels (spiral-like or convoluted lines/circles), linear (fine lines), curved (comma-like lines) and serpiginous
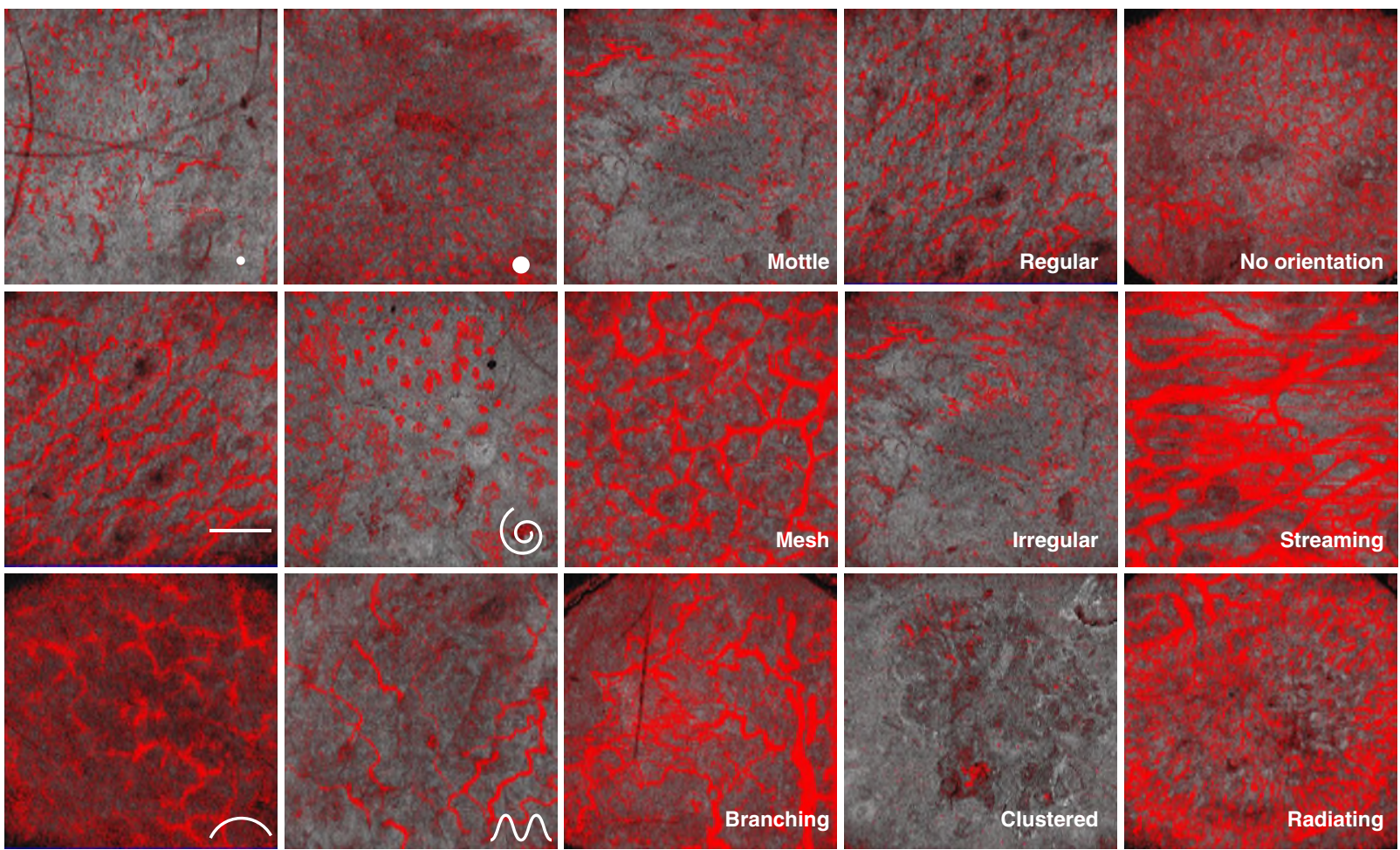

Figure 4 Correlations of schematic illustrations of the different shapes with real D-OCT images are presented. 
vessels. Further descriptors can be added at S-parameters. The dots, blobs and coiled vessel shapes could be described as 'mottled' in case they are distributed in limited but sharply demarcated areas; linear vessels can be defined as a 'mesh' when they are interconnected and generate a reticular structure; 'linear', 'curved, 'serpiginous' vessels can present branching that in case can be defined as 'arborizing' if a progressive decrease in the thickness is observed in subsequent branches, or 'bulging' if enlarged buds originate from the main structure (Figs. 3 and 4).

\section{Cross-sectional image parameters}

Two major vessel patterns can be detected and described in cross-sectional images when present: spikes (needle-shaped narrow peaks of vessels vertically directed towards the surface) and columns (brighter rectangular-shaped structures with an upper horizontal sharply demarcated border and often with a 'waterfall'-like signal stretching into the dermis (corresponding to a technical artefact). ${ }^{7}$

\section{Discussion}

Very recently, first investigations have reported the applicability of D-OCT for the evaluation of vascular patterns in vivo including tumour angiogenesis ${ }^{7}$ and functional blood vessel dilatation/ constriction. ${ }^{8,10}$ In contrast to established techniques such as dermoscopy and reflectance confocal microscopy, D-OCT allows the analysis of microvasculature to a depth of at least $500 \mu \mathrm{m}$ and the $3 \mathrm{D}$ reconstruction of vascular networks. Although first results seem promising, a standardized terminology is required to secure that the results of ongoing research can be communicated in a valid and reproducible manner. Clear descriptors are of outermost importance before further research on skin diseases is conducted. Therefore, we held an expert meeting to define common features and patterns of vessel distribution as depicted by D-OCT. Following this, we provide the reader with recommendations for optimal image acquisition and interpretation of D-OCT images using a series of descriptors for the different vascular shapes and their arrangement. Shapes and patterns have been described using geometrical definitions and avoiding metaphoric terms in the aim to increase objectiveness. Furthermore, we suggest five criteria (the 'D-parameters': 'depth', 'density', 'diameter', 'direction' and 'distribution') that need to be consid- ered when analysing D-OCT in a detailed and systematic way, in order to produce a more reproducible description and to provide identification of parameters depicting the global vascular status of the imaged area. In our case review, these descriptors seemed to delineate distinct patterns and a different intensity of increased vascularization in various skin tumours. Further studies are required in order to test the reproducibility of these parameters and their capability to distinguish different biologi$\mathrm{cal} /$ pathological conditions, such as tumour pattern of growth and aggressiveness. D-OCT opens the possibility to in vivo visualize the microvasculature of the skin. A basic terminology would be of help to standardize definitions and generate more reproducible and comparable studies.

\section{References}

1 Sattler E, Kästle R, Welzel J. Optical coherence tomography in dermatology. J Biomed Opt 2013; 18: 061224.

2 Ulrich M, Themstrup L, de Carvalho N et al. Dynamic optical coherence tomography in dermatology. Dermatology 2016; 232: 298-311.

3 Ulrich $\mathrm{M}$, von Braunmuehl T, Kurzen $\mathrm{H}$ et al. The sensitivity and specificity of optical coherence tomography for the assisted diagnosis of nonpigmented basal cell carcinoma: an observational study. Br J Dermatol 2015; 173: 428-435.

4 Hussain AA, Themstrup L, Jemec GB. Optical coherence tomography in the diagnosis of basal cell carcinoma. Arch Dermatol Res 2015; 307: 1-10.

5 Mogensen M, Thrane L, Joergensen TM, Andersen PE, Jemec GB. Optical coherence tomography for imaging of skin and skin diseases. Semin Cutan Med Surg 2009; 28: 196-202.

6 Schuh S, Kaestle R, Sattler E, Welzel J. Comparison of different optical coherence tomography devices for diagnosis of non-melanoma skin cancer. Skin Res Technol 2016; 22: 395-405.

7 De Carvalho N, Ciardo S, Cesinaro A et al. In vivo micro-angiography by means of speckle-variance optical coherence tomography (SV-OCT) is able to detect microscopic vascular changes in naevus to melanoma transition. J Eur Acad Dermatol Venereol 2016; 30: e67-e68.

8 Themstrup L, Ciardo S, Manfredi M et al. In vivo, micro-morphological vascular changes induced by topical brimonidine studied by dynamic optical coherence tomography. J Eur Acad Dermatol Venerol 2016; 30: 974-979.

9 Manfredi M, Grana C, Pellacani G. Skin Surface Reconstruction and 3D Vessels Segmentation in Speckle Variance Optical Coherence Tomography. In Proceedings of the 11th Joint Conference on Computer Vision, Imaging and Computer Graphics Theory and Applications, Volume 4: VISAPP, 2016, 234-240. https://doi.org/10.5220/0005758702340240, ISBN 978-989-758-175-5.

10 Themstrup L, Welzel J, Ciardo S et al. Validation of Dynamic optical coherence tomography for non-invasive, in vivo microcirculation imaging of the skin. Microvasc Res 2016; 107: 97-105. 